\title{
Design, synthesis and anti-influenza virus activity of furan-substituted spirothiazolidinones
}

Çağla Begüm Apaydın ${ }^{1 *}$, Merve Tansuyu ${ }^{1}$, Zafer Cesur ${ }^{1}$, Lieve Naesens ${ }^{2}$, Füsun Göktaş ${ }^{1}$

${ }^{1}$ Faculty of Pharmacy, Department of Pharmaceutical Chemistry, Istanbul University, Istanbul, Turkey

${ }^{2}$ Rega Institute, KU Leuven, Department of Microbiology, Immunology and Transplantation, B-3000, Leuven, Belgium

*Corresponding author: Çağla Begüm Apaydın, Faculty of Pharmacy, Department of Pharmaceutical Chemistry, Istanbul University, 34126, Istanbul, Fatih, Turkey.

Phone: 021244000 00/ 13462. E-mail: cagla.apaydin@istanbul.edu.tr

\begin{abstract}
A new series of $N$-(3-oxo-1-thia-4-azaspiro[4.5]decan-4-yl)carboxamides have been designed, synthesized and evaluated as antiviral agents. The compounds were prepared by condensation of 2-methylfuran-3-carbohydrazide, appropriate carbonyl compounds and sulfanyl acids. The new molecules were characterized by IR, ${ }^{1} \mathrm{H}-\mathrm{NMR},{ }^{13} \mathrm{C}-\mathrm{NMR}$, mass spectrometry and elemental analysis. Six analogues proved to be active against influenza A/H3N2 virus, the two most protent analogues, $\mathbf{3 c}$ and $\mathbf{3 d}$, having an $\mathrm{EC}_{50}$ value of about $1 \mu \mathrm{M}$. These findings help to define the SAR of spirothiazolidinone-based inhibitors of the influenza virus membrane fusion process.
\end{abstract}

Keywords: synthesis, antiviral activity, furan, spirothiazolidinone, influenza virus 


\section{Introduction}

Influenza is an acute respiratory infection caused by human influenza A, B and C viruses which belong to the Orthomyxoviridae family. Characteristic symptoms are: sudden fever, muscle pain, weakness, chills, headache and dry cough [1]. In some patients, this can evolve into acute viral or secondary bacterial pneumonia. The annually occurring influenza epidemics are explained by antigenic drift of the virus [2]. Besides, influenza A viruses with a zoonotic origin cause sporadic pandemics with high morbidity and mortality. In the last 100 years, four influenza pandemics have occurred: the H1N1 Spanish influenza in 1918; H2N2 Asian influenza in 1957; H3N2 Hong Kong influenza in 1968; and swine-origin H1N1 influenza in 2009 [3].

Influenza A, B and C viruses exhibit differences in the nucleoprotein and matrix proteins. The further subtyping of influenza A virus is based on two envelope glycoproteins: hemagglutinin (HA) and neuraminidase (NA). At the moment, the treatment of influenza relies on three classes of FDA-approved antiviral drugs (Figure 1), targeting the M2 proton channel, neuraminidase enzyme or viral polymerase complex [4]. The influenza A virus-specific M2 inhibitors amantadine and rimantadine are no longer recommended, due to widespread viral resistance against these agents $[5,6]$. The neuraminidase inhibitors oseltamivir, zanamivir and peramivir inhibit influenza A and B viruses. In the past few years, two polymerase inhibitors, i.e. favipiravir and baloxavir marboxil, have been approved in a few countries. Favipiravir inhibits the RNA-dependent RNA polymerase function of the viral PB1 protein [7], while baloxavir marboxil targets the cap-dependent endonuclease activity of the PA protein [8]. The latter drug shows superior clinical efficacy [9], which seems threatened by growing concerns on emergence of baloxavir-resistant mutant viruses [10]. Arbidol (umifenovir) has been reported to inhibit hemagglutinin (HA)-mediated fusion by preventing the conformational change of HA at low pH. An indole-based small molecule, arbidol, has been licensed in Russia and China for prophylaxis and treatment of influenza and other viral respiratory infections (Figure 1). It is in clinical influenza trials in the USA [11,12]. 


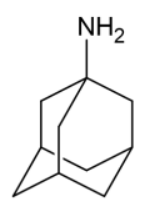

Amantadine

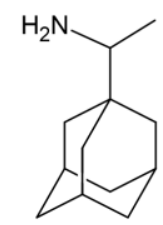

Rimantadine

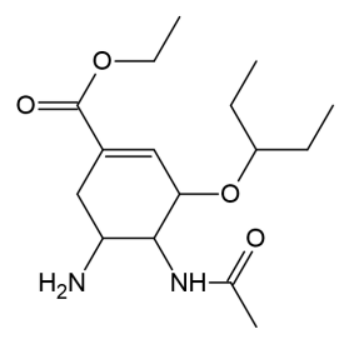

Oseltamivir<smiles>CC(=O)NC1C(N=C(N)N)C=C(C(=O)O)OC1C(O)C(O)CO</smiles>

Zanamivir<smiles>CCC(CC)C(NC(C)=O)C1C(NC(=N)N)CC(C(=O)O)C1O</smiles>

Peramivir<smiles>NC(=O)c1nc(F)cnc1O</smiles>

Favipiravir<smiles>COC(=O)OCOC1C(=O)CCN2C1C(=O)N1CCOCC1N2C1c2ccccc2SCc2c1ccc(F)c2F</smiles>

Baloxavir marboxil<smiles>CCOC(=O)c1c(CSc2ccccc2)n(C)c2cc(Br)c(O)c(CN(C)C)c12</smiles>

Arbidol

Figure 1: Chemical structures of FDA-approved antiviral drugs and arbidol

About ten years ago, our research team identified a structurally unique class of spiro compounds, exerting strong inhibition of the HA-mediated membrane fusion process [13]. Subsequently, several series of structural analogues were synthesized, which enabled quite detailed insight in the structure-activity relationship (SAR) (Figure 2) [13-18]. The lead compound is encoded as $\mathbf{4 c}$ in [Ref.13]; [6-methyl- $N$-(2,8-dimethyl-3-oxo-1-thia-4azaspiro[4.5]dec-4-yl)imidazo[2,1-b][1,3]thiazole-5-carboxamide] (Figure 2). The general structure consists of an aromatic ring that is linked, via an amide bridge, to a non-aromatic spirocyclic system. In this spirothiazolidinone part, the methyl substituents at positions 2 and 8 proved to be important for antiviral activity. Mechanistically, these molecules act by preventing the low pH-induced conformational change of HA, that occurs after uptake of the virus in endosomes and that is crucial for membrane fusion and release of the viral genome. Resistance 
studies [13] and HA docking analyses [18] provided an explanation for the HA binding mode and H3 HA-subtype specificity of these fusion inhibitors. In the present study, we report the design and synthesis of a new series of spirothiazolidinone derivatives carrrying a 3furancarboxamide moiety. The newly synthesized compounds were evaluated for in vitro antiviral activity against influenza $\mathrm{A}$ and $\mathrm{B}$ viruses.

$\underline{R}$<smiles>CCc1c(C)nc2sccn12</smiles>

4c

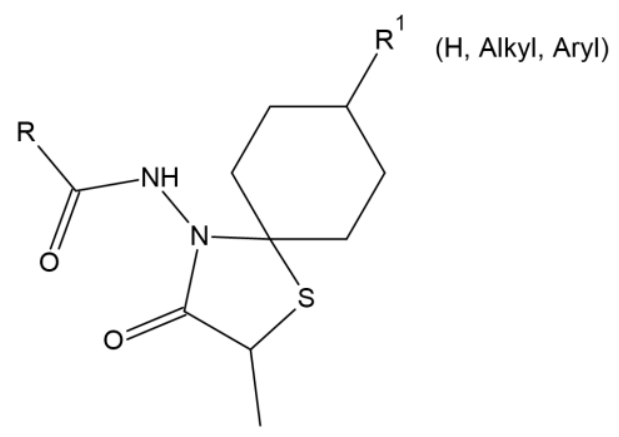<smiles>Cc1cc2ccccc2cc1O</smiles><smiles>Cc1[nH]c2ccc(Cl)cc2c1C</smiles><smiles>CCC12CC3CC(CC(C3)C1)C2</smiles>

Figure 2: Chemical structures of previously reported spirothiazolidinone inhibitors of influenza virus fusion [13-18].

\section{Results and discussion}

\subsection{Chemistry}

The synthetic pathway for the preparation of compounds $(\mathbf{2}, \mathbf{3})$ is shown in Scheme 1. Refluxing a mixture of hydrazide (1) and the appropriate cyclic ketone with sulfanylacetic acid or 2sulfanylpropanoic acid in dry toluene using a Dean-Stark aparatus, afforded the target compounds $\mathbf{2}, \mathbf{3}$. The structures of the new compounds were confirmed by microanalysis, IR, ${ }^{1} \mathrm{H}-\mathrm{NMR},{ }^{13} \mathrm{C}-\mathrm{NMR}$ and mass spectrometry.

The IR spectra of the 2a-f and 3a-f derivatives have N-H stretching bands at $3427-3224 \mathrm{~cm}^{-1}$. Observation of new lactam $\mathrm{C}=\mathrm{O}$ bands $\left(1715-1685 \mathrm{~cm}^{-1}\right)$ characteristic for such structures besides $\mathrm{C}=\mathrm{O}$ amide bands (1681-1658 $\left.\mathrm{cm}^{-1}\right)$ in the IR spectra of compounds $\mathbf{2}$ and $\mathbf{3}$ supported the targeted cyclization. In the ${ }^{1} \mathrm{H}-\mathrm{NMR}$ spectra, the N-H protons appeared in the region of 10.09-8.55 ppm. The C2-H protons of 2a-f were observed 3.58-3.64 ppm as singlets, while C2- 
$\mathrm{H}$ protons of 3a-f resonated at 3.75-3.79 ppm as quartets. Also, ${ }^{13} \mathrm{C}-\mathrm{NMR}$ spectra of $\mathbf{2} \mathbf{b}$ and $\mathbf{3 a} \mathbf{a}-$ f confirmed formation of the expected spirothiazolidinones.

Further spectral details are available in the experimental section.
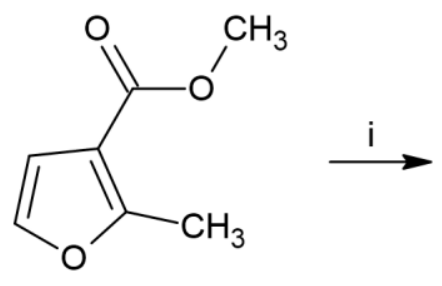

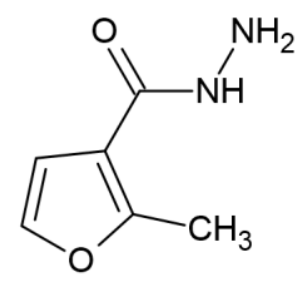

1

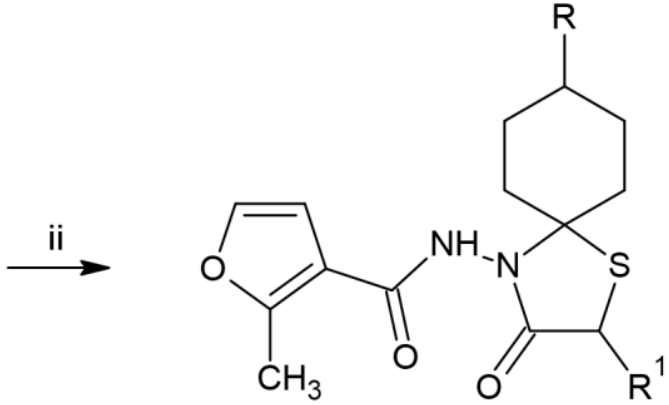

2 (a-f), 3 (a-f)

$17-75 \%$

\begin{tabular}{cccc|cccc}
\hline Compound & $\mathbf{R}$ & $\mathbf{R}^{\mathbf{1}}$ & Yield (\%) & Compound & $\mathbf{R}$ & $\mathbf{R}^{\mathbf{1}}$ & Yield (\%) \\
\hline $\mathbf{2 a}$ & $\mathrm{H}$ & $\mathrm{H}$ & 68 & $\mathbf{3 a}$ & $\mathrm{H}$ & $\mathrm{CH}_{3}$ & 45 \\
$\mathbf{2 b}$ & $\mathrm{CH}_{3}$ & $\mathrm{H}$ & 71 & $\mathbf{3 b}$ & $\mathrm{CH}_{3}$ & $\mathrm{CH}_{3}$ & 17 \\
$\mathbf{2 c}$ & $\mathrm{C}_{2} \mathrm{H}_{5}$ & $\mathrm{H}$ & 74 & $\mathbf{3 c}$ & $\mathrm{C}_{2} \mathrm{H}_{5}$ & $\mathrm{CH}_{3}$ & 52 \\
$\mathbf{2 d}$ & $\mathrm{C}_{3} \mathrm{H}_{7}$ & $\mathrm{H}$ & $\mathbf{7 7}$ & $\mathbf{3 d}$ & $\mathrm{C}_{3} \mathrm{H}_{7}$ & $\mathrm{CH}_{3}$ & 40 \\
$\mathbf{2 e}$ & $\mathrm{C}^{2}\left(\mathrm{CH}_{3}\right)_{3}$ & $\mathrm{H}$ & 68 & $\mathbf{3 e}$ & $\mathrm{C}_{\left(\mathrm{CH}_{3}\right)_{3}}$ & $\mathrm{CH}_{3}$ & 56 \\
$\mathbf{2 f}$ & $\mathrm{C}_{6} \mathrm{H}_{5}$ & $\mathrm{H}$ & 75 & $\mathbf{3 f}$ & $\mathrm{C}_{6} \mathrm{H}_{5}$ & $\mathrm{CH}_{3}$ & 60 \\
\hline
\end{tabular}

Scheme 1: Synthesis of compounds 2, 3. Reagents and conditions: (i) hydrazine hydrate, ethanol, reflux, 16 h; (ii) (non)substituted ketone, sulfanylacetic acid or 2-sulfanylpropanoic acid, toluene, 12-16 h.

\subsection{Biological activity}

The new compounds were evaluated in Madin-Darby canine kidney (MDCK) cells infected with influenza $\mathrm{A} / \mathrm{H} 1 \mathrm{~N} 1, \mathrm{~A} / \mathrm{H} 3 \mathrm{~N} 2$ or $\mathrm{B}$ virus. The antiviral procedure estimated inhibition of virus-induced cytopathic effect (CPE), using microscopy and MTS cell viability assay. These two methods also allowed to determine compound cytotoxicity in mock-infected cell cultures. Most molecules were devoid of toxicity at $100 \mu \mathrm{M}$, the highest concentration tested. Six analogues, i.e. $\mathbf{2 b}, \mathbf{2 d}, \mathbf{2 e}, \mathbf{3 b}, \mathbf{3 c}$ and $\mathbf{3 d}$ were found to inhibit influenza A/H3N2 virus (Table 1). Compounds $\mathbf{3 c}$ and $\mathbf{3 d}$ demonstrated the highest inhibitory activity, with antiviral $\mathrm{EC}_{50}$ values of $0.95 \mu \mathrm{M}$ and $0.93 \mu \mathrm{M}$, respectively (values based on the MTS readout). These analogues bear a methyl group at position $2\left(\mathrm{R}^{1}\right)$, besides an ethyl (3c) or propyl (3d) substituent at position $8(\mathrm{R})$. This SAR fully agrees with earlier analyses on the spirothiazolidinone 
compounds [13]. An important new insight was that the anti-influenza virus activity is unchanged when imidazothiazole system in $\mathbf{4 c}$ is replaced by a furan moiety. With regard to the spiro part, substitution at position 2 or 8 seems essential. A methyl group at position 2 (in type 3 ) is obviously required for higher activity since the analogues $\mathbf{2 b}, \mathbf{2 c}$ and $\mathbf{2 d}$ lacking this group are less active than their 2-methylated counterparts $\mathbf{3 b}, \mathbf{3} \mathbf{c}$ and $\mathbf{3 d}$ (except $\mathbf{2 e}$ and $\mathbf{3 e}$ ). Regarding position 8 of the spiro ring, a larger alkyl group is preferable over a smaller alkyl substituent for higher activity (i.e., n-propyl in 3d> ethyl in 3c> methyl in 3b). Interestingly, 3c and 3d were about 8 -fold more active than our initial lead molecule (compound $\mathbf{4 c}$ in Table 1), which was instrumental to identify the antiviral mechanism of action of the spirothiazolidinone derivatives [16]. This means that the 2-methylfuran moiety of $\mathbf{3 c}$ and $\mathbf{3 d}$ is superior to the imidazo[2,1-b]thiazol part of $\mathbf{4 c}$. Besides, also these new furan analogues exhibit A/H3N2specificity, since no antiviral activity was seen against influenza A/H1N1 virus and influenza $\mathrm{B}$ virus.

Table 1: Anti-influenza virus activity of compounds $\mathbf{2 a - f}$ and $\mathbf{3 a - f}$.

\begin{tabular}{|c|c|c|c|c|c|c|c|c|c|c|}
\hline \multirow[t]{3}{*}{ Compound $^{\mathrm{a}}$} & \multirow[t]{3}{*}{$\mathrm{R}$} & \multirow[t]{3}{*}{$\mathrm{R}^{1}$} & \multicolumn{6}{|c|}{ Antiviral $\mathrm{EC}_{50} \mathrm{~b}(\mu \mathrm{M})$} & \multicolumn{2}{|c|}{$\begin{array}{c}\text { Cytotoxicity }^{\mathrm{c}} \\
(\mu \mathrm{M})\end{array}$} \\
\hline & & & \multicolumn{2}{|c|}{$\mathrm{A} / \mathrm{H} 1 \mathrm{~N} 1$} & \multicolumn{2}{|c|}{$\mathrm{A} / \mathrm{H} 3 \mathrm{~N} 2$} & \multicolumn{2}{|c|}{ Influenza B } & \multirow[t]{2}{*}{ MCC } & \multirow[t]{2}{*}{$\mathrm{CC}_{50}$} \\
\hline & & & $\mathrm{CPE}$ & MTS & $\mathrm{CPE}$ & MTS & $\mathrm{CPE}$ & MTS & & \\
\hline $2 \mathbf{a}$ & $\mathrm{H}$ & $\mathrm{H}$ & $>100$ & $>100$ & $>100$ & $>100$ & $>100$ & $>100$ & $>100$ & $>100$ \\
\hline $2 \mathbf{b}$ & $\mathrm{CH}_{3}$ & $\mathrm{H}$ & $>100$ & $>100$ & $>100$ & 44 & $>100$ & $>100$ & $>100$ & $>100$ \\
\hline $2 c$ & $\mathrm{C}_{2} \mathrm{H}_{5}$ & $\mathrm{H}$ & $>100$ & $>100$ & $>100$ & $>100$ & $>100$ & $>100$ & $\geq 20$ & 45 \\
\hline 2d & $\mathrm{C}_{3} \mathrm{H}_{7}$ & $\mathrm{H}$ & $>100$ & $>100$ & 21 & 6.6 & $>100$ & $>100$ & $>100$ & $>100$ \\
\hline $2 e$ & $\mathrm{C}\left(\mathrm{CH}_{3}\right)_{3}$ & $\mathrm{H}$ & $>100$ & $>100$ & 7.9 & 3.5 & $>100$ & $>100$ & $\geq 100$ & $>100$ \\
\hline $2 f$ & $\mathrm{C}_{6} \mathrm{H}_{5}$ & $\mathrm{H}$ & $>100$ & $>100$ & $>100$ & $>100$ & $>100$ & $>100$ & 100 & 43 \\
\hline 3a & $\mathrm{H}$ & $\mathrm{CH}_{3}$ & $>100$ & $>100$ & $>100$ & $>100$ & $>100$ & $>100$ & $>100$ & $>100$ \\
\hline $3 \mathbf{b}$ & $\mathrm{CH}_{3}$ & $\mathrm{CH}_{3}$ & $>100$ & $>100$ & 12 & 8.5 & $>100$ & $>100$ & $>100$ & $>100$ \\
\hline $3 c$ & $\mathrm{C}_{2} \mathrm{H}_{5}$ & $\mathrm{CH}_{3}$ & $>100$ & $>100$ & 1.4 & 0.95 & $>100$ & $>100$ & 100 & $>100$ \\
\hline 3d & $\mathrm{C}_{3} \mathrm{H}_{7}$ & $\mathrm{CH}_{3}$ & $>100$ & $>100$ & 0.80 & 0.93 & $>100$ & $>100$ & 60 & 43 \\
\hline $3 e$ & $\mathrm{C}\left(\mathrm{CH}_{3}\right)_{3}$ & $\mathrm{CH}_{3}$ & $>100$ & $>100$ & $>100$ & $>100$ & $>100$ & $>100$ & $>100$ & $>100$ \\
\hline $3 f$ & $\mathrm{C}_{6} \mathrm{H}_{5}$ & $\mathrm{CH}_{3}$ & $>100$ & $>100$ & $>100$ & $>100$ & $>100$ & $>100$ & $>100$ & $>100$ \\
\hline $\mathbf{4 c}[13]$ & $\mathrm{CH}_{3}$ & $\mathrm{CH}_{3}$ & $>100$ & $>100$ & 6.8 & 9.0 & $>100$ & $>100$ & $>100$ & $>100$ \\
\hline ZAN & - & - & 0.44 & 0.48 & 0.043 & 0.012 & 0.072 & 0.063 & $>100$ & $>100$ \\
\hline RBV & - & - & 8.9 & 8.4 & 8.9 & 10 & 8.3 & 8.1 & $\geq 20$ & $>100$ \\
\hline AMT & - & - & 58 & 75 & 0.80 & 0.70 & $>500$ & $>500$ & $\geq 500$ & $>500$ \\
\hline
\end{tabular}




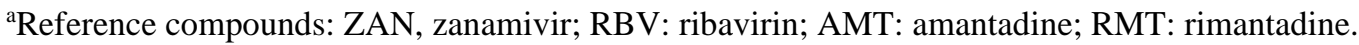

${ }^{\mathrm{b}} \mathrm{EC}_{50}: 50 \%$ effective concentration giving $50 \%$ inhibition of virus-induced cytopathicity, as estimated by microscopic inspection of the CPE (left columns) or by the MTS cell viability assay (right columns). Virus strains: A/PR/8/34 (A/H1N1); A/HK/7/87 (A/H3N2 and B/HK/5/72.

${ }^{\mathrm{c}} \mathrm{MCC}$ : minimum cytotoxic concentration, based on microscopic inspection of cell morphology; CC50: $50 \%$ cytotoxic concentration, based on the MTS cell viability assay.

\section{Conclusion}

A new series of $N$-(3-oxo-1-thia-4-azaspiro[4.5]decan-4-yl)carboxamides were obtained by using a three-component one-pot cyclocondensation method. The structures of the new compounds were characterized and confirmed by spectrometric methods and elemental analysis. Analogues 3c and 3d had superior activity against influenza A/H3N2 virus, consistent with the importance of the 2- and 8-substituents in the spirothiazolidinone system. In addition, this new series shows that the aromatic part of this class of H3 HA-specific fusion inhibitors tolerates much variation.

\section{Experimental}

\subsection{Materials}

Chemicals were obtained from Merck and Aldrich. Melting points (mp) were determined on a Buchi B-540 capillary melting point apparatus in open capillaries and uncorrected. IR spectra were recorded in $\mathrm{KBr}$ discs on a Shimadzu IR Affinity-1 FTIR and ${ }^{1} \mathrm{H}-\mathrm{NMR}$ (DMSO-d 6 )

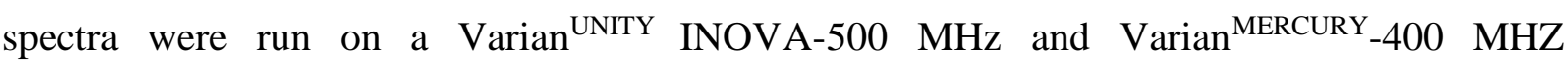
spectrophotometers. Chemical shifts are reported as $\delta(\mathrm{ppm})$ relative to TMS as internal standard and coupling constants $(J)$ are given in hertz $(\mathrm{Hz})$. Microanalyses were performed on a Thermo Finnigan Flash EA 1112 elemental analyzer. ESI/MS were determined on Finnigan LCQ Advantage Max spectrophotometer (sp: spirothiazolidinone, frn: furan, ax: axial, eq: equatorial).

\subsection{Chemical synthesis}

\subsubsection{2-Methylfuran-3-carbohydrazide (1)}

$0.26 \mathrm{~mol}$ hydrazine hydrate $(98 \%)$ was added to a solution of $0.026 \mathrm{~mol}$ methyl 2-methylfuran3-carboxylate in $12 \mathrm{ml}$ alcohol (96\%) and the mixture was heated under reflux for $16 \mathrm{~h}$. The resulting residue was allowed to stand overnight. The solid thus obtained was washed with ice water, dried and used without purification. 


\section{General procedure for the synthesis of $(2,3)$}

A solution of $1(0.005 \mathrm{~mol})$ and appropriate ketone $(0.01 \mathrm{~mol})$ in $30 \mathrm{ml}$ of dried toluene were refluxed for $2 \mathrm{~h}$, using a Dean Stark water separator. After $2 \mathrm{~h}$, sulfanylacetic acid or 2sulfanylpropanoic acid $(1.5 \mathrm{ml})$ was added and the mixture was refluxed during 12-16 $\mathrm{h}$. Toluene was evaporated in vасио. The residue was neutralized with saturated sodium bicarbonate and allowed to solidify. The crude product was filtered and recrystallized from appropriate solvent or solvent mixtures.

\subsection{2. $N$-(3-oxo-1-thia-4-azaspiro[4.5]decan-4-yl)-2-methylfuran-3-carboxamide (2a)}

Yield: 68\%. mp: 206-208 ${ }^{\circ} \mathrm{C}$; IR (KBr) $v\left(\mathrm{~cm}^{-1}\right)$ : $3224(\mathrm{~N}-\mathrm{H}), 1693(\mathrm{C}=\mathrm{O}), 1662(\mathrm{NHC}=\mathrm{O})$.

${ }^{1} \mathrm{H}-\mathrm{NMR}$ (DMSO-d 6 /500MHz): 1.01-1.05 (1H, m, sp-8ax-H), 1.40-1.43 (2H, m, sp-7ax-H ve sp$\left.9_{\mathrm{ax}}-\mathrm{H}\right), 1.52-1.54\left(1 \mathrm{H}, \mathrm{d},{ }^{3} \mathrm{~J}=13 \mathrm{~Hz}, \mathrm{sp}-8_{\mathrm{eq}}-\mathrm{H}\right), 1.70-1.82\left(6 \mathrm{H}, \mathrm{m}, \mathrm{sp}-6-\mathrm{H}, \mathrm{sp}-7_{\mathrm{eq}}-\mathrm{H}\right.$ sp- $9_{\mathrm{eq}}-\mathrm{H}$ and sp-10-H), 3.29 (3H, s, frn-2- $\left.\mathrm{CH}_{3}\right), 3.58(2 \mathrm{H}, \mathrm{s}, \mathrm{sp}-2-\mathrm{H}), 6.93\left(1 \mathrm{H}, \mathrm{d},{ }^{3} \mathrm{~J}=2 \mathrm{~Hz}\right.$, frn-4-H), 7.57 $\left(1 \mathrm{H}, \mathrm{d},{ }^{3} J=2 \mathrm{~Hz}\right.$, frn-5-H), $10.01(1 \mathrm{H}, \mathrm{s}, \mathrm{NH})$. Anal. calcd. for $\mathrm{C}_{15} \mathrm{H}_{20} \mathrm{~N}_{2} \mathrm{O}_{3} \mathrm{~S}$ (294.36) C: 57.12, H: 6.16, N: 9.52. Found C: 56.69, H: 6.04, N: 9.72.

\subsection{3. $N$-(8-methyl-3-oxo-1-thia-4-azaspiro[4.5]decan-4-yl)-2-methylfuran-3- carboxamide (2b)}

Yield: $71 \%$. mp: $180-183{ }^{\circ} \mathrm{C}$; IR $(\mathrm{KBr}) \cup\left(\mathrm{cm}^{-1}\right): 3304(\mathrm{~N}-\mathrm{H}), 1701(\mathrm{C}=\mathrm{O}), 1678(\mathrm{NHC}=\mathrm{O})$. ${ }^{1} \mathrm{H}-\mathrm{NMR}$ (DMSO-d 6 /500MHz): $0.85\left(3 \mathrm{H}, \mathrm{d},{ }^{3} \mathrm{~J}=7 \mathrm{~Hz}, \mathrm{sp}-8-\mathrm{CH}_{3}\right), 1.12-1.28(3 \mathrm{H}, \mathrm{m}, \mathrm{sp}-7 \mathrm{ax}-\mathrm{H}$, sp-8-H and sp-9 $\left.9_{\mathrm{ax}}-\mathrm{H}\right), 1.66-1.80$ (6H, m, sp-6-H, sp-7 eq $^{-H}$, sp- $9_{\mathrm{eq}}-\mathrm{H}$ and sp-10-H), 3.29 (3H, s, frn-2- $\left.\mathrm{CH}_{3}\right), 3.59$ (2H, s, sp-2-H), $6.92\left(1 \mathrm{H}, \mathrm{d},{ }^{3} \mathrm{~J}=2 \mathrm{~Hz}\right.$, frn-4-H), $7.57\left(1 \mathrm{H}, \mathrm{d},{ }^{3} \mathrm{~J}=2 \mathrm{~Hz}\right.$, frn-5$\mathrm{H}), 10.00(1 \mathrm{H}, \mathrm{s}, \mathrm{NH}) .{ }^{13} \mathrm{C}-\mathrm{NMR}$ (proton decoupled) (DMSO-d $6 / 125 \mathrm{MHz}$ ): 14.00 (frn-C2$\mathrm{CH}_{3}$ ), 22.55 (sp-C8-CH3), 28.59 (sp-C8), 31.17 (sp-C7 and sp-C9), 32.07 (sp-C6 and sp-C10), 37.63 (sp-C2), 72.83 (sp-C5), 109.67 (frn-C4), 113.97 (frn-C3), 141.81 (frn-C5), 158.22 (frnC2), 163.16 (NHCO), 168.53 (sp-C3). Anal. calcd. for $\mathrm{C}_{15} \mathrm{H}_{20} \mathrm{~N}_{2} \mathrm{O}_{3} \mathrm{~S}$ (308.39) C: 58.42, H:6.54, N: 9.08. Found C: 58.54, H: 6.45, N: 9.28.

\subsection{4. $N$-(8-ethyl-3-oxo-1-thia-4-azaspiro[4.5]decan-4-yl)-2-methylfuran-3-carboxamide} (2c)

Yield: $74 \%$. mp: $168-170{ }^{\circ} \mathrm{C}$; IR (KBr) $v\left(\mathrm{~cm}^{-1}\right): 3427(\mathrm{~N}-\mathrm{H}), 1685(\mathrm{C}=\mathrm{O}), 1658(\mathrm{NHC}=\mathrm{O})$. ${ }^{1} \mathrm{H}-\mathrm{NMR}$ (DMSO-d 6 /500MHz): $0.82\left(3 \mathrm{H}, \mathrm{t},{ }^{3} \mathrm{~J}=7 \mathrm{~Hz}, \mathrm{sp}-8-\mathrm{CH}_{2} \mathbf{C H}_{3}\right), 1.00-1.30(5 \mathrm{H}, \mathrm{m}$, sp7 ax $-\mathrm{H}, \mathrm{sp}-8-\mathrm{H}, \mathrm{sp}-9_{\mathrm{ax}}-\mathrm{H}$ and sp-8- $\left.\mathrm{CH}_{2}\right), 1.60-2.50$ (6H, m, sp-6-H, sp- $7_{\mathrm{eq}}-\mathrm{H}$ and sp- $9_{\mathrm{eq}}-\mathrm{H}, \mathrm{sp}-10-$ H), $3.30\left(3 \mathrm{H}, \mathrm{s}\right.$, frn-2-CH $\mathrm{CH}_{3}, 3.58(2 \mathrm{H}, \mathrm{s}, \mathrm{sp}-2-\mathrm{H}), 6.92\left(1 \mathrm{H}, \mathrm{d},{ }^{3} \mathrm{~J}=2 \mathrm{~Hz}\right.$, frn-4-H), $7.57(1 \mathrm{H}, \mathrm{d}$, 
${ }^{3} J=2 \mathrm{~Hz}$, frn-5-H), $10.01(1 \mathrm{H}, \mathrm{s}, \mathrm{NH})$. Anal. calcd. for $\mathrm{C}_{16} \mathrm{H}_{22} \mathrm{~N}_{2} \mathrm{O}_{3} \mathrm{~S}$ (322.42) C: 59.60, H: 6.88, N: 8.69. Found C: 59.39, H: 6.77, N: 8.97.

\subsection{5. $N$-(3-oxo-8-propyl-1-thia-4-azaspiro[4.5]decan-4-yl)-2-methylfuran-3-carboxamide} (2d)

Yield: 77\%. mp: $205-20{ }^{\circ} \mathrm{C}$; IR (KBr) v $\left(\mathrm{cm}^{-1}\right): 3234(\mathrm{~N}-\mathrm{H}), 1699(\mathrm{C}=\mathrm{O}), 1670(\mathrm{NHC}=\mathrm{O})$. ${ }^{1} \mathrm{H}-\mathrm{NMR}$ (DMSO-d $\left.6 / 500 \mathrm{MHz}\right): 0.83\left(3 \mathrm{H}, \mathrm{t},{ }^{3} \mathrm{~J}=7 \mathrm{~Hz}, \mathrm{sp}-8-\mathrm{CH}_{2} \mathrm{CH}_{2} \mathbf{C H}_{3}\right), 1.11-1.20(5 \mathrm{H}, \mathrm{m}$, sp-8- $\mathbf{C H}_{2} \mathrm{CH}_{2} \mathrm{CH}_{3}$, sp-7 ${ }_{\mathrm{ax}}-\mathrm{H}$, sp- $9_{\mathrm{ax}}-\mathrm{H}$ and sp-8-H), 1.23-1.27 (2H, m, sp-8- $\left.\mathrm{CH}_{2} \mathbf{C H}_{2} \mathrm{CH}_{3}\right)$, 1.72-1.82 (6H, m, sp-6-H, sp-7 eq $^{-H}$ and sp- 9 eq $\left.-\mathrm{H}, \mathrm{sp}-10-\mathrm{H}\right), 3.29(3 \mathrm{H}, \mathrm{s}$, frn-2-CH$), 3.58(2 \mathrm{H}$, s, sp-2-H), $6.92\left(1 \mathrm{H}, \mathrm{d},{ }^{3} J=2 \mathrm{~Hz}\right.$, frn-4-H), $7.57\left(1 \mathrm{H}, \mathrm{d},{ }^{3} J=2 \mathrm{~Hz}\right.$, frn-5-H), $10.01(1 \mathrm{H}, \mathrm{s}, \mathrm{NH})$. Anal. calcd. for $\mathrm{C}_{17} \mathrm{H}_{24} \mathrm{~N}_{2} \mathrm{O}_{3} \mathrm{~S}$ (336.44) C: 60.69, H: 7.19, N: 8.33. Found C: 60.89, H: 7.27, N: 8.52 .

\subsection{6. $N$-(8-tert-butyl-3-oxo-1-thia-4-azaspiro[4.5]dec-4-yl)-2-methylfuran-3-carboxamide} (2e)

Yield: 68\%. mp: $180-182{ }^{\circ} \mathrm{C}$; IR (KBr) $v\left(\mathrm{~cm}^{-1}\right)$ : $3236(\mathrm{~N}-\mathrm{H}), 1701(\mathrm{C}=\mathrm{O}), 1670(\mathrm{NHC}=\mathrm{O})$. ${ }^{1} \mathrm{H}-\mathrm{NMR}$ (DMSO-d 6 /500MHz): $0.81\left(9 \mathrm{H}, \mathrm{s}, \mathrm{sp}-8-\mathrm{C}\left(\mathrm{CH}_{3}\right)_{3}\right), 0.85-0.93\left(1 \mathrm{H}, \mathrm{m}, \mathrm{C}_{8}-\mathrm{H}\right), 1.18-1.22$ $\left(2 \mathrm{H}, \mathrm{m}, \mathrm{sp}-7_{\mathrm{ax}}-\mathrm{H}\right.$ and sp- $\left.9_{\mathrm{ax}}-\mathrm{H}\right), 1.70-2.20\left(6 \mathrm{H}, \mathrm{m}, \mathrm{sp}-6-\mathrm{H}, \mathrm{sp}-7_{\mathrm{eq}}-\mathrm{H}\right.$ and sp- $\left.9_{\mathrm{eq}}-\mathrm{H}, \mathrm{sp}-10-\mathrm{H}\right)$, $3.30\left(3 \mathrm{H}, \mathrm{s}\right.$, frn-2- $\left.\mathrm{CH}_{3}\right), 3.58(2 \mathrm{H}, \mathrm{s}, \mathrm{sp}-2-\mathrm{H}), 6.92\left(1 \mathrm{H}, \mathrm{d},{ }^{3} \mathrm{~J}=2 \mathrm{~Hz}\right.$, frn-4-H), $7.57\left(1 \mathrm{H}, \mathrm{d},{ }^{3} J=\right.$ $2 \mathrm{~Hz}$, frn-5-H), $10.02(1 \mathrm{H}, \mathrm{s}, \mathrm{NH})$. Anal. calcd. for $\mathrm{C}_{18} \mathrm{H}_{26} \mathrm{~N}_{2} \mathrm{O}_{3} \mathrm{~S} . \mathrm{H}_{2} \mathrm{O}$ (368.47) C: 58.67, H: 7.66, N: 7.99. Found C: 58.50, H: 7.46, N: 7.86.

\subsection{7. $\quad N$-(3-oxo-8-phenyl-1-thia-4-azaspiro[4.5]dec-4-yl)-2-methylfuran-3-carboxamide} (2f)

Yield: 75\%. mp: $224-226{ }^{\circ} \mathrm{C}$; IR (KBr) $v\left(\mathrm{~cm}^{-1}\right): 3296(\mathrm{~N}-\mathrm{H}), 1703(\mathrm{C}=\mathrm{O}), 1681(\mathrm{NHC}=\mathrm{O})$. ${ }^{1} \mathrm{H}-\mathrm{NMR}$ (DMSO-d 6 /400MHz): 1.64-1.67 (2H, m, sp-7ax-H and sp-9ax-H), 1.85-2.20 (6H, m, sp-6-H, sp-7eq-H, sp-9eq-H and sp-10-H), 2.46-2.48 (1H, m, sp-8-H and DMSO-d 6 ), 3.31 (3H, s, frn-2- $\mathrm{CH}_{3}$ and DMSO- $\left.\mathrm{H}_{2} \mathrm{O}\right), 3.64(2 \mathrm{H}, \mathrm{s}, \mathrm{sp}-2-\mathrm{H}), 6.96\left(1 \mathrm{H}, \mathrm{d},{ }^{3} \mathrm{~J}=2 \mathrm{~Hz}\right.$, frn-4-H), 7.14-7.32 $\left(5 \mathrm{H}, \mathrm{m}\right.$, phenyl H), $7.60\left(1 \mathrm{H}, \mathrm{d},{ }^{3} J=2 \mathrm{~Hz}\right.$, frn-5-H), $10.11(1 \mathrm{H}, \mathrm{s}, \mathrm{NH})$.

\subsection{8. $N$-(2-methyl-3-oxo-1-thia-4-azaspiro[4.5]dec-4-yl)-2-methylfuran-3-carboxamide} (3a)

Yield: 45\%. mp: $173-175^{\circ} \mathrm{C}$; IR (KBr) v $\left(\mathrm{cm}^{-1}\right)$ : $3294(\mathrm{~N}-\mathrm{H}), 1708(\mathrm{C}=\mathrm{O}), 1660(\mathrm{NHC}=\mathrm{O}) .{ }^{1} \mathrm{H}-$ NMR (CDCl $3 / 500 \mathrm{MHz}): 0.93-1.04\left(1 \mathrm{H}, \mathrm{m}, \mathrm{sp}-8_{\mathrm{ax}}-\mathrm{H}\right), 1.36-1.51\left(2 \mathrm{H}, \mathrm{m}, \mathrm{sp}-7_{\mathrm{ax}}-\mathrm{H}\right.$ and sp- $9_{\mathrm{ax}}-$ H), $1.49\left(3 \mathrm{H}, \mathrm{d},{ }^{3} \mathrm{~J}=7 \mathrm{~Hz}, \mathrm{sp}-2-\mathrm{CH}_{3}\right), 1.51-1.58\left(1 \mathrm{H}, \mathrm{m}, \mathrm{sp}-8_{\mathrm{eq}}-\mathrm{H}\right), 1.65-1.72\left(2 \mathrm{H}, \mathrm{m}, \mathrm{sp}-7_{\mathrm{eq}}-\mathrm{H}\right.$ and sp-9 $\left.9_{\mathrm{eq}} \mathrm{H}\right), 1.73\left(1 \mathrm{H}, \mathrm{d},{ }^{3} J=13 \mathrm{~Hz}, \mathrm{sp}-10_{\mathrm{eq}}-\mathrm{H}\right), 1.74\left(1 \mathrm{H}, \mathrm{d},{ }^{2} J=13 \mathrm{~Hz}, \mathrm{sp}-6_{\mathrm{eq}}-\mathrm{H}\right), 1.82(1 \mathrm{H}$, 
$\left.\mathrm{d},{ }^{2} J=13 \mathrm{~Hz}, \mathrm{sp}-10_{\mathrm{eq}}-\mathrm{H}\right), 1.85-2.00\left(1 \mathrm{H}, \mathrm{m}, \mathrm{sp}-6_{\mathrm{ax}}-\mathrm{H}\right), 2.39(3 \mathrm{H}, \mathrm{s}$, frn-2-CH$), 3.79\left(1 \mathrm{H}, \mathrm{q},{ }^{3} J=7\right.$ $\mathrm{Hz}, \mathrm{SCH}), 6.65\left(1 \mathrm{H}, \mathrm{d},{ }^{3} J=2 \mathrm{~Hz}\right.$, frn-4-H), 7.07 (1H, d, ${ }^{3} \mathrm{~J}=2 \mathrm{~Hz}$, frn-5-H), 8.85 (1H, s, NH).

${ }^{13} \mathrm{C}-\mathrm{NMR}$ (DEPT) $\left(\mathrm{CDCl}_{3} / 125 \mathrm{MHz}\right)$ : 13.79 (frn-C2-CH $), 20.12$ (sp-C2-CH3), 23.25 and 23.73 (sp-C7 and sp-C9), 24.59 (sp-C8), 37.82 (sp-C2), 37.63 and 38.80 (sp-C6 and sp-C10), 108.75 (frn-C4), 140.28 (frn-C5). ESI (-) MS m/z (\%) : 307 ([M-H]', 38.8), 263 (18.82), 235 (46.94), 219 (100), 141 (75.00). Anal. calcd. for $\mathrm{C}_{15} \mathrm{H}_{20} \mathrm{~N}_{2} \mathrm{O}_{3} \mathrm{~S}$ (308.39) C: 58.42, H: 6.54, N: 9.08. Found C: 58.52, H: 6.19, N: 9.02.

\subsection{9.}

$N$-(2,8-dimethyl-3-oxo-1-thia-4-azaspiro[4.5]dec-4-yl)-2-methylfuran-3-

\section{carboxamide (3b)}

Yield: $17 \%$. mp: $168-169^{\circ} \mathrm{C}$; IR (KBr) v $\left(\mathrm{cm}^{-1}\right): 3234(\mathrm{~N}-\mathrm{H}), 1708(\mathrm{C}=\mathrm{O}), 1673(\mathrm{NHC}=\mathrm{O}) .{ }^{1} \mathrm{H}-$ NMR ( $\left.\mathrm{CDCl}_{3} / 500 \mathrm{MHz}\right): 0.82\left(3 \mathrm{H}, \mathrm{d},{ }^{3} \mathrm{~J}=6 \mathrm{~Hz}, \mathrm{sp}-8-\mathrm{CH}_{3}\right), 1.08-1.26$ (3H, m, sp-7ax-H, sp-9ax$\mathrm{H}$ and sp-8-H), 1.49 (3H, d, $\left.{ }^{3} \mathrm{~J}=7 \mathrm{~Hz}, \mathrm{sp}-2-\mathrm{CH}_{3}\right), 1.60-1.68$ (2H, m, sp-7eq-H and sp-9eq-H), $1.73\left(1 \mathrm{H}, \mathrm{d},{ }^{2} J=13 \mathrm{~Hz}, \mathrm{sp}-6 \mathrm{eq}-\mathrm{H}\right), 1.76-1.85$ (2H, m, sp-10eq-H and sp-10ax-H), 1.92-1.96 (1H, m, sp-6ax-H), 2.39 (3H, s, frn-2- $\left.\mathrm{CH}_{3}\right), 3.79\left(1 \mathrm{H}, \mathrm{q},{ }^{3} \mathrm{~J}=7 \mathrm{~Hz}, \mathrm{SCH}\right), 6.64\left(1 \mathrm{H}, \mathrm{d},{ }^{3} \mathrm{~J}=2 \mathrm{~Hz}\right.$, frn4-H), 7.08 (1H, d, ${ }^{3} J=2 \mathrm{~Hz}$, frn-5-H), 8.87 (1H, s, NH). ${ }^{13} \mathrm{C}-\mathrm{NMR}$ (DEPT) $\left(\mathrm{CDCl}_{3} / 125 \mathrm{MHz}\right)$ : 12.55 (frn-C2- $\mathrm{CH}_{3}$ ), 18.88 (sp-C2- $\mathrm{CH}_{3}$ ), 20.79 (sp-C8-CH ), 30.02 (sp-C8), 30.44 and 30.95 (sp-C7 and sp-C9), 36.60 (sp-C2), 36.12 and 37.30 (sp-C6 and sp-C10), 107.52 (frn-C4), 139.03 (frn-C5). ESI (-) MS m/z (\%): 321 ([M-H]', 14.90), 277 (12.15), 249 (40.83); 233 (100), 141 (70.46). Anal. calcd. for $\mathrm{C}_{16} \mathrm{H}_{22} \mathrm{~N}_{2} \mathrm{O}_{3} \mathrm{~S}$ (322.42) C: 59.60, H: 6.88, N: 8.69. Found C: 59.54, H: $6.84, \mathrm{~N}: 8.55$.

\subsubsection{0. $\quad N$-(8-ethyl-2-methyl-3-oxo-1-thia-4-azaspiro[4.5]dec-4-yl)-2-methylfuran-3- carboxyamide (3c)}

Yield: 52\%. mp: $160-162^{\circ} \mathrm{C}$; IR (KBr) $v\left(\mathrm{~cm}^{-1}\right): 3227(\mathrm{~N}-\mathrm{H}), 1705(\mathrm{C}=\mathrm{O}), 1678(\mathrm{NHC}=\mathrm{O}) .{ }^{1} \mathrm{H}-$ NMR ( $\left.\mathrm{CDCl}_{3} / 500 \mathrm{MHz}\right): 0.78\left(3 \mathrm{H}, \mathrm{t},{ }^{3} J=7 \mathrm{~Hz}, \mathrm{sp}-8-\mathrm{CH}_{2} \mathbf{C H} 3\right), 0.93-1.03$ (1H, m, sp-8-H), 1.03$1.28\left(2 \mathrm{H}, \mathrm{m}, \mathrm{sp}-7 \mathrm{ax}-\mathrm{H}\right.$ and sp-9ax-H), $1.15\left(2 \mathrm{H}, \mathrm{q},{ }^{3} \mathrm{~J}=7 \mathrm{~Hz}, \mathrm{sp}-8-\mathrm{CH}_{2}\right), 1.49\left(3 \mathrm{H}, \mathrm{d},{ }^{3} \mathrm{~J}=7 \mathrm{~Hz}\right.$, sp-2- $\left.\mathrm{CH}_{3}\right), 1.68-1.81(4 \mathrm{H}, \mathrm{m}, \mathrm{sp}-6 \mathrm{eq}-\mathrm{H}, \mathrm{sp}-10 \mathrm{ax}-\mathrm{H}, \mathrm{sp}-7 \mathrm{eq}-\mathrm{H}$ and sp-9eq-H), $1.83(1 \mathrm{H}, \mathrm{d}$, $\left.{ }^{2} J=13 \mathrm{~Hz}, \mathrm{sp}-10 \mathrm{eq}-\mathrm{H}\right), 1.90-1.96(1 \mathrm{H}, \mathrm{m}, \mathrm{sp}-6 \mathrm{ax}-\mathrm{H}), 2.39\left(3 \mathrm{H}, \mathrm{s}, \mathrm{frn}-2-\mathrm{CH}_{3}\right), 3.79\left(1 \mathrm{H}, \mathrm{q},{ }^{3} J=7\right.$ $\mathrm{Hz}, \mathrm{SCH}), 6.64\left(1 \mathrm{H}, \mathrm{d},{ }^{3} \mathrm{~J}=2 \mathrm{~Hz}\right.$, frn-4-H), 7.07 (1H, d, ${ }^{3} \mathrm{~J}=2 \mathrm{~Hz}$, frn-5-H), 8.89 (1H, s, NH). ${ }^{13} \mathrm{C}-\mathrm{NMR}$ (APT) $\left(\mathrm{CDCl}_{3} / 125 \mathrm{MHz}\right): 10.43$ (sp-C8- $\left.\mathrm{CH}_{2} \mathbf{C H}_{3}\right), 12.54$ (frn-C2-CH 3 ), 18.91 (sp$\mathrm{C} 2-\mathrm{CH}_{3}$ ), 28.10 (sp-C8-CH $), 28.05$ and 28.56 (sp-C7 and sp-C9), 36.61 (sp-C2), 36.66 (spC8), 36.13 and 37.30 (sp-C6 and sp-C10), 71.60 (sp-C5), 107.54 (frn-C4), 111.62 (frn-C3), 139.02 (frn-C5), 158.07 (frn-C2), 162.05 (NHCO), 172.12 (sp-C3). ESI (-) MS m/z (\%): 335 
([M-H]', 18.82), 291 (26.80), 263 (48.48), 247 (100), 141 (43.28). Anal. calcd. for $\mathrm{C}_{17} \mathrm{H}_{24} \mathrm{~N}_{2} \mathrm{O}_{3} \mathrm{~S}$. $0.5 \mathrm{H}_{2} \mathrm{O}(345.45) \mathrm{C}: 59.10, \mathrm{H}: 7.29, \mathrm{~N}: 8.11$. Found C: 59.68; H: 7.17; N: 8.23.

4.2.11. $\quad N$-(2-methyl-3-oxo-8-propyl-1-thia-4-azaspiro[4.5]dec-4-yl)-2-methylfuran-3carboxamide (3d)

Yield: $40 \%$. mp: $172-174^{\circ} \mathrm{C}$; IR (KBr) v $\left(\mathrm{cm}^{-1}\right): 3234(\mathrm{~N}-\mathrm{H}), 1704(\mathrm{C}=\mathrm{O}), 1675(\mathrm{NHC}=\mathrm{O}) .{ }^{1} \mathrm{H}-$ NMR ( $\left.\mathrm{CDCl}_{3} / 500 \mathrm{MHz}\right): 0.79$ and $0.81\left(3 \mathrm{H}, \mathrm{tt},{ }^{3} \mathrm{~J}=7 \mathrm{~Hz}, \mathrm{sp}-8-\mathrm{CH}_{2} \mathrm{CH}_{2} \mathbf{C H}_{3}\right), 1.10$ (2H, m, spC8-CH2), 1.05-1.27 (3H, m, sp-7ax-H, sp-9-ax-H and sp-8-H), $1.20\left(2 \mathrm{H}, \mathrm{m}, \mathrm{sp}-8-\mathrm{CH}_{2} \mathrm{CH}_{2}\right)$, $1.49\left(3 \mathrm{H}, \mathrm{d},{ }^{3} \mathrm{~J}=7 \mathrm{~Hz}, \mathrm{sp}-2-\mathrm{CH}_{3}\right), 1.66-1.73(2 \mathrm{H}, \mathrm{m}, \mathrm{sp}-7 \mathrm{eq}-\mathrm{H}$ and sp-9eq-H), $1.73-1.81(2 \mathrm{H}, \mathrm{m}$, sp-6eq-H and sp-10ax-H), 1.83 (1H, d, ${ }^{2} J=13 \mathrm{~Hz}$ sp-10eq-H), 1.88-1.97 (1H, m, sp-6ax-H), $2.41(3 \mathrm{H}, \mathrm{s}$, frn-2-CH 3$), 3.79\left(1 \mathrm{H}, \mathrm{q},{ }^{3} J=7 \mathrm{~Hz}, \mathrm{SCH}\right), 6.60\left(1 \mathrm{H}, \mathrm{d},{ }^{3} \mathrm{~J}=2 \mathrm{~Hz}\right.$, frn-4-H), $7.10(1 \mathrm{H}$, d, ${ }^{3} \mathrm{~J}=2 \mathrm{~Hz}$, frn-5-H), $8.56(1 \mathrm{H}, \mathrm{s}, \mathrm{NH}) .{ }^{13} \mathrm{C}-\mathrm{NMR}$ (APT) $\left(\mathrm{CDCl}_{3} / 125 \mathrm{MHz}\right): 13.78$ (frn-C2$\mathrm{CH} 3$ ), 14.42 (sp-C8- $\mathrm{CH}_{2} \mathrm{CH}_{2} \mathrm{CH}_{3}$ ), 20.14 (sp-C2-CH3), 20.20 (sp-C8- $\mathrm{CH}_{2} \mathrm{CH}_{2}$ ), 29.66 and 30.16 (sp-C7 and sp-C9), 35.82 (sp-C8), 37.84 (sp-C2), 37.39 and 38.55 (sp-C6 and sp-C10), 38.92 (sp-C8-CH2), 72.81 (sp-C5), 108.76 (frn-C4), 112.84 (frn-C3), 140.27 (frn-C5), 159.32 (frn-C2), 163.30 (NHCO), 173.33 (sp-C3). ESI (-) MS m/z (\%): 349 ([M-H]', 18.32), 305 (14.27), 277 (28.17), 261 (100), 141 (35.56). Anal. calcd. for $\mathrm{C}_{18} \mathrm{H}_{26} \mathrm{~N}_{2} \mathrm{O}_{3}$ S. $0.25 \mathrm{H}_{2} \mathrm{O}$ (354.97) C: $60.90, \mathrm{H}: 7.52, \mathrm{~N}: 7.89$. Found C: 60,81, H: 7.40, N: 7.52 .

\subsubsection{2. $N$-(8-tert-buthyl-2-methyl-3-oxo-1-thia-4-azaspiro[4.5]dec-4-yl)-2-methylfuran-3- carboxamide (3e)}

Yield: 56\%. mp: $226-228^{\circ} \mathrm{C}$; IR (KBr) v $\left(\mathrm{cm}^{-1}\right): 3319(\mathrm{~N}-\mathrm{H}), 1715(\mathrm{C}=\mathrm{O}), 1671(\mathrm{NHC}=\mathrm{O}) .{ }^{1} \mathrm{H}-$

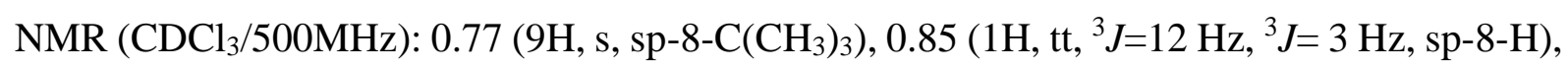
1.15-1.32 (2H, m, sp-7ax-H and sp-9ax-H), 1.50 (3H, d, ${ }^{3} \mathrm{~J}=7 \mathrm{~Hz}$, sty-2-CH 3$), 1.69-1.77(2 \mathrm{H}$, m, sp-7eq-H and sp-9eq-H), 1.77-1.85 (2H, m, sp-6eq-H and sp-10ax-H), 1.85-1.98 (2H, m, sp6ax-H and sp-10eq-H), $2.42(3 \mathrm{H}, \mathrm{s}$, frn-2-CH$), 3.79\left(1 \mathrm{H}, \mathrm{q},{ }^{3} \mathrm{~J}=7 \mathrm{~Hz}, \mathrm{SCH}\right), 6.60\left(1 \mathrm{H}, \mathrm{d},{ }^{3} J=2\right.$ $\mathrm{Hz}$, frn-4-H), $7.10\left(1 \mathrm{H}, \mathrm{d},{ }^{3} \mathrm{~J}=2 \mathrm{~Hz}\right.$, frn-5-H), 8.55 (1H, s, NH). ${ }^{13} \mathrm{C}-\mathrm{NMR}(\mathrm{HMBC})\left(\mathrm{CDCl}_{3} / 125\right.$ MHz): 12.58 (frn-C2-CH CH $_{3} 19.00$ (sp-C2-CH3), 22.92 and 23.37 (sp-C7 and sp-C9), 26.45 (sp$\mathrm{C} 8-\mathrm{C}\left(\mathrm{CH}_{3}\right)_{3}$ ), 31.23 (sp-C8-C), 36.61 (sp-C2), 36.56 and 37.68 (sp-C6 and sp-C10), 45.35 (spC8), 71.37 (sp-C5), 107.47 (frn-C4), 111.63 (frn-C3), 139.13 (frn-C5), 158.12 (frn-C2), 162.12 (NHCO), 171.98 (sp-C3). ${ }^{13} \mathrm{C}-\mathrm{NMR}$ (DEPT) $\left(\mathrm{CDCl}_{3} / 125 \mathrm{MHz}\right): 12.57$ (frn-C2-CH 3 ), 19.00 (sp-C2- $\mathrm{CH}_{3}$ ), 22.91 and 23.36 (sp-C7 and sp-C9), 26.44 (sp-C8-C( $\left.\left.\mathrm{CH}_{3}\right)_{3}\right), 36.60$ (sp-C2), 36.54 and 37.67 (sp-C6 and sp-C10), 45.34 (sp-C8), 107.45 (frn-C4), 139.12 (frn-C5). ESI (-) MS m/z (\%) : 363 ([M-H]', 8.52), 319 (11.26), 291 (48.27), 275 (100), 141 (33.87). Anal. calcd. for $\mathrm{C}_{19} \mathrm{H}_{28} \mathrm{~N}_{2} \mathrm{O}_{3} \mathrm{~S}$ (364.50) C: 62.61, H: 7.74, N: 7.69. Found C: 62.76, H: 7.46, N: 7.16. 


\subsubsection{3. $\quad N$-(8-phenyl-2-methyl-3-oxo-1-thia-4-azaspiro[4.5]dec-4-yl)-2-methylfuran-3-}

carboxamide (3f)

Yield: 60\%. mp: 218-220 ${ }^{\circ}$; IR (KBr) v $\left(\mathrm{cm}^{-1}\right): 3233(\mathrm{~N}-\mathrm{H}), 1701(\mathrm{C}=\mathrm{O}), 1670(\mathrm{NHC}=\mathrm{O}) .{ }^{1} \mathrm{H}-$ NMR $\left(\mathrm{CDCl}_{3} / 500 \mathrm{MHz}\right): 1.53\left(3 \mathrm{H}, \mathrm{d},{ }^{3} \mathrm{~J}=7 \mathrm{~Hz}, \mathrm{sp}-2-\mathrm{CH}_{3}\right), 1.65-1.83(2 \mathrm{H}, \mathrm{m}, \mathrm{sp}-7 \mathrm{ax}-\mathrm{H}$ and sp9ax-H), 1.85-1.94 (3H, m, sp-6eq-H, sp-7eq-H and sp-9eq-H), 1,94-2,04 (2H, m, sp-10eq-H and sp-10ax-H), 2.10-2.18 (1H, m, sp-6ax-H), $2.38\left(1 \mathrm{H}, \mathrm{tt},{ }^{3} J=12 \mathrm{~Hz},{ }^{3} J=3 \mathrm{~Hz}, \mathrm{sp}-8-\mathrm{H}\right), 2.42$ $\left(3 \mathrm{H}, \mathrm{s}\right.$, frn-2- $\left.\mathrm{CH}_{3}\right), 3.75\left(1 \mathrm{H}, \mathrm{q},{ }^{3} J=7 \mathrm{~Hz}, \mathrm{SCH}\right), 6.66\left(1 \mathrm{H}, \mathrm{d},{ }^{3} \mathrm{~J}=2 \mathrm{~Hz}\right.$, frn-4-H), 7.08-7.17 (4H, $\mathrm{m}$, phenyl-2-H, phenyl-6-H, phenyl-4-H and frn-5-H), 7.19-7.27 (2H, m, phenyl-3-H and phenyl-5-H), 8.79 (1H, s, NH). ${ }^{13} \mathrm{C}-\mathrm{NMR}$ (DEPT) $\left(\mathrm{CDCl}_{3} / 125 \mathrm{MHz}\right): 12.59$ (frn-C2-CH ${ }_{3}$ ), 18.94 (sp-C2-CH ${ }_{3}$ ), 29.53 and 30.06 (sp-C7 and sp-C9), 36.73 (sp-C2), 36.45 and 37.59 (sp-C6 and sp-C10), 41.45 (sp-C8), 107.53 (frn-C4), 125.32 (phenyl-C4), 125.69 (phenyl-C2 and phenyl-C6), 127.45 (phenyl-C3 ve phenyl-C5), 139.09 (frn-C5). ESI (-) MS m/z (\%) : 383 ([M$\left.\mathrm{H}]^{-}, 16.05\right), 339$ (13.70), 311 (38.89), 295 (100), 141 (28.16). Anal. calcd. for $\mathrm{C}_{21} \mathrm{H}_{24} \mathrm{~N}_{2} \mathrm{O}_{3} \mathrm{~S}$ (384.49) C: 65.60, H: 6.29, N: 7.29. Found C: 65.39, H: 6.17, N: 8.59.

\subsection{Biological methods to assess anti-influenza virus activity}

The molecules were evaluated in a cytopathic effect (CPE) reduction assay in influenza virusinfected Madin Darby canine kidney (MDCK) cells [19]. As control compounds, zanamivir, ribavirin, amantadine and rimantadine were included. MDCK cells were seeded into 96-well plates at 7,500 cells per well. On the next day, serial compound dilutions were added to the cells, together with influenza virus [multiplicity of infection: fifty $50 \%$ cell culture infective doses $\left(\mathrm{CCID}_{50}\right)$ per well]. The mock-infected plate received the same compound dilutions but medium instead of virus. After three days incubation at $35^{\circ} \mathrm{C}$, microscopy was performed to determine antiviral activity, expressed as the concentration producing 50\% inhibition of virusinduced $\mathrm{CPE}$ (50\% effective concentration $\left[\mathrm{EC}_{50}\right]$ ), as well as compound cytotoxicity, expressed as the concentration causing minimal changes in cell morphology (MCC). Next, the 3-(4,5-dimethylthiazol-2-yl)-5-(3-carboxymethoxyphenyl)-2-(4-sulfophenyl)-2H-tetrazolium (MTS) reagent was added [CellTiter 96 AQueous One Solution Cell Proliferation Assay from Promega]. After $4 \mathrm{~h}$ incubation, absorbance was measured at $490 \mathrm{~nm}$ and the spectrophotometric data were used to calculate the $\mathrm{EC}_{50}$ and $50 \%$ cytotoxic concentration $\left(\mathrm{CC}_{50}\right)$. 


\section{Acknowledgements}

This work was supported in part by a Research Fund from Istanbul University (Project number 4216). L.N. would like to thank the team of Leentje Persoons for fine technical assistance.

\section{Conflict of interest}

The authors have declared no conflict of interest.

\section{References}

[1] F. Krammer, G.J.D. Smith, R.A.M. Fouchier, M. Peiris, K. Kedzierska, P.C. Doherty, P. Palese, M.L. Shaw, J. Treanor, R.G. Webster, A. García-Sastre, Influenza, Nat. Rev. Dis. Primers. 4 (2018) 1-21. https://doi.org/10.1038/s41572-018-0002-y.

[2] J.S. Long, B. Mistry, S.M. Haslam, W.S. Barclay, Host and viral determinants of influenza A virus species specificity, Nat. Rev. Microbiol. 17 (2019) 67-81. https://doi.org/10.1038/s41579-018-0115-z.

[3] G. Neumann, T. Noda, Y. Kawaoka, Emergence and pandemic potential of swineorigin H1N1 influenza virus, Nature 459 (2009) 931-939.

https://doi.org/10.1038/nature08157.

[4] L. Naesens, A. Stevaert, E. Vanderlinden, Antiviral therapies on the horizon for influenza, Curr. Opin. Pharmacol. 30 (2016) 106-115. https://doi.org/10.1016/j.coph.2016.08.003.

[5] A. Moscona, Medical Management of Influenza Infection, Annu. Rev. Med. 59 (2008) 397-413. https://doi.org/10.1146/annurev.med.59.061506.213121.

[6] V.M. Deyde, X. Xu, R.A. Bright, M. Shaw, C.B. Smith, Y. Zhang, Y. Shu, L.V Gubareva, N.J. Cox, A.I. Klimov, Surveillance of Resistance to Adamantanes among Influenza A (H3N2) and A (H1N1) Viruses Isolated Worldwide, J. Infect. Dis. 196 (2007) 249-257. https://doi.org/10.1086/518936.

[7] Y. Furuta, B.B. Gowen, K. Takahashi, K. Shiraki, D.F. Smee, D.L. Barnard, Favipiravir (T-705), a novel viral RNA polymerase inhibitor, Antiviral. Res. 100 (2013) 446-454. https://doi.org/10.1016/j.antiviral.2013.09.015.

[8] S. Omoto, V. Speranzini, T. Hashimoto, T. Noshi, H. Yamaguchi, M. Kawai, K. Kawaguchi, T. Uehara, T. Shishido, A. Naito, S. Cusack, Characterization of influenza virus variants induced by treatment with the endonuclease inhibitor baloxavir marboxil, Sci. Rep. (2018) 1-15. https://doi.org/10.1038/s41598-018-27890-4.

[9] F.G. Hayden, N. Sugaya, N. Hirotsu, N. Lee, M.D. de Jong, A.C. Hurt, T. Ishida, H. Sekino, K. Yamada, S. Portsmouth, K. Kawaguchi, T. Shishido, M. Arai, K. Tsuchiya, T. Uehara, A. Watanabe, Baloxavir Marboxil Investigators G, Baloxavir marboxil for uncomplicated influenza in adults and adolescents, N. Engl. J. Med. 379 (2018) 913923. https://doi.org/10.1056/NEJMoa1716197. 
[10] T. Uehara, F.G. Hayden, K. Kawaguchi, S. Omoto, A.C. Hurt, M.D. De Jong, N. Hirotsu, N. Sugaya, N. Lee, K. Baba, T. Shishido, K. Tsuchiya, S. Portsmouth, H. Kida, Treatment-Emergent Influenza Variant Viruses With Reduced Baloxavir Susceptibility: Impact on Clinical and Virologic Outcomes in Uncomplicated Influenza, J. Infect. Dis. 221 (2019) 346-355. https://doi.org/10.1093/infdis/jiz244.

[11] F. Li, C. Ma, J. Wang, Inhibitors Targeting the Influenza Virus Hemagglutinin, Curr. Med. Chem. 22 (2015) 1-22. https://doi.org/10.2174/0929867322666150227153919.

[12] Ç.B. Apaydın, G. Çınar, G. Cihan-Üstündağ, Small-molecule Antiviral Agents in Ongoing Clinical Trials for COVID-19, Curr. Drug Targets 22 (2021) 1-20. https://doi.org/10.2174/1389450122666210215112150.

[13] E. Vanderlinden, F. Göktaş, Z. Cesur, M. Froeyen, M.L. Reed, C.J. Russell, N. Cesur, L. Naesens, Novel Inhibitors of Influenza Virus Fusion: Structure-Activity Relationship and Interaction with the Viral Hemagglutinin, J. Virol. 84 (2010) 42774288. https://doi.org/10.1128/JVI.02325-09.

[14] F. Göktas, E. Vanderlinden, L. Naesens, N. Cesur, Z. Cesur, Microwave assisted synthesis and anti-influenza virus activity of 1-adamantyl substituted N-(1-thia-4azaspiro[4.5]decan-4-yl)carboxamide derivatives, Bioorg. Med. Chem. 20 (2012) 7155-7159. https://doi.org/10.1016/j.bmc.2012.09.064.

[15] F. Göktaş, E. Vanderlinden, L. Naesens, Z. Cesur, P. Taş, Synthesis and structureactivity relationship of N-(3-oxo-1-thia-4-azaspiro[4.5] decan-4-yl)carboxamide inhibitors of influenza virus hemagglutinin mediated fusion, Phosphorus Sulfur Silicon Relat. Elem. 190 (2015) 1075-1087. https://doi.org/10.1080/10426507.2014.965819.

[16] F. Göktaş, M. Özbil, N. Cesur, E. Vanderlinden, L. Naesens, Z. Cesur, Novel N-(1thia-4-azaspiro[4.5]decan-4-yl)carboxamide derivatives as potent and selective influenza virus fusion inhibitors, Arch. Pharm. (Weinheim) 352 (2019) e1900028. https://doi.org/10.1002/ardp.201900028.

[17] A. Kocabalkanlı, G. Cihan-Ustündağ, L. Naesens, E. Matarac1-Kara, M. Nassozi, G. Çapan, Diclofenac-Based Hydrazones and Spirothiazolidinones: Synthesis, Characterization, and Antimicrobial Properties, Arch. Pharm. (Weinheim) 350 (2017) e1700010. https://doi.org/10.1002/ardp.201700010.

[18] G. Cihan-Ustündağ, M. Zopun, E. Vanderlinden, E. Ozkirimli, L. Persoons, G. Capan, L. Naesens, Superior inhibition of influenza virus hemagglutinin-mediated fusion by indole-substituted spirothiazolidinones, Bioorg. Med. Chem. 28 (2020) 115130. https://doi.org/10.1016/j.bmc.2019.115130.

[19] P. Vrijens, S. Noppen, T. Boogaerts, E. Vanstreels, R. Ronca, P. Chiodelli, M. Laporte, E. Vanderlinden, S. Liekens, A. Stevaert, L. Naesens, Influenza virus entry via the GM3 ganglioside-mediated platelet-derived growth factor receptor beta signalling pathway, J. Gen. Virol. 100 (2019) 583-601. https://doi.org/10.1099/jgv.0.001235. 